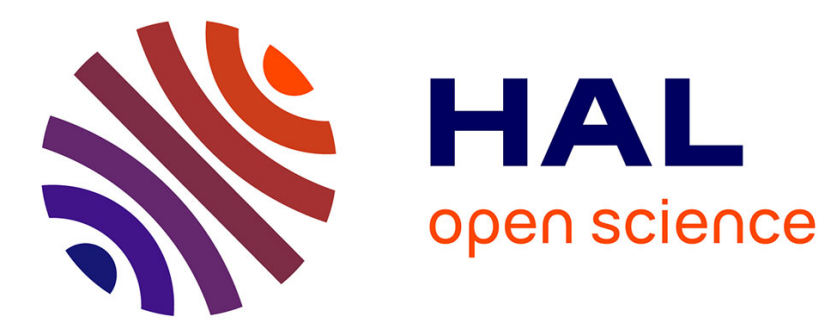

\title{
Spectromètre SISAM à détection synchrone
}

P. Pinson, J.P. Guillotin

\section{To cite this version:}

P. Pinson, J.P. Guillotin. Spectromètre SISAM à détection synchrone. Revue de Physique Appliquée, 1972, 7 (4), pp.335-337. 10.1051/rphysap:0197200704033500 . jpa-00243636

\section{HAL Id: jpa-00243636 https://hal.science/jpa-00243636}

Submitted on 1 Jan 1972

HAL is a multi-disciplinary open access archive for the deposit and dissemination of scientific research documents, whether they are published or not. The documents may come from teaching and research institutions in France or abroad, or from public or private research centers.
L'archive ouverte pluridisciplinaire HAL, est destinée au dépôt et à la diffusion de documents scientifiques de niveau recherche, publiés ou non, émanant des établissements d'enseignement et de recherche français ou étrangers, des laboratoires publics ou privés. 
Classification

Physics Abstracts

08.45

\title{
SPECTROMẼTRE SISAM A DÉTECTION SYNCHRONE
}

\author{
P. PINSON et J. P. GUILLOTIN (*) \\ Laboratoire d'Infrarouge, Equipe de Recherche Associée au CNRS \\ Université de Paris VI, Campus d'Orsay, Bât. 350 (91) Orsay
}

(Reçu le 15 mai 1972)

\begin{abstract}
Résumé. - Un système de modulation cohérente du faisceau lumineux dans un spectromètre SISAM a été étudié. Il permet une détection synchrone du signal. Des spectres infrarouges sont présentés qui montrent un gain notable sur le rapport signal/bruit et sur le produit temps $\times$ résolution de l'appareil.
\end{abstract}

Abstract. - A method for coherent modulation of optical beam in a SISAM spectrometer has been studied. It allows a synchronous detection of the signal. Infrared spectra are shown with a considerable increase in signal/noise ratio and in time $\times$ resolution product of the spectrometer.

Le SISAM (Spectromètre Interférentiel à Sélection par l'Amplitude de Modulation) a été inventé par P. Connes en 1957 [1], [2]. Plusieurs spectromètres ont été construits selon la conception originale. Récemment des études ont été entreprises au Laboratoire pour améliorer la résolution intrinsèque de l'appareil [3], [4]. Deux montages, l'un permettant un double passage de la lumière sur les réseaux et l'autre réalisant un réglage asymétrique, permettent de doubler la résolution. La combinaison des deux montages conduit à un appareil dont la résolution est quatre fois plus élevée.

Dans le SISAM original (Fig. 1a) la modulation est produite par l'oscillation de la lame compensatrice $\mathrm{C}$.

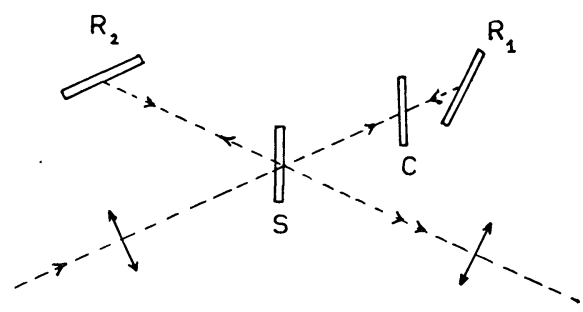

FIG. $1 a$.

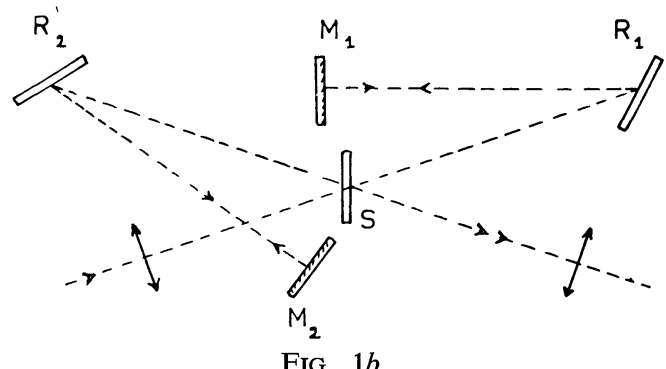

FIG. 1. - a) SISAM original. b) SISAM à double passage.

(*) Actuellement détaché de la Société anonyme de Télécommunication.
La différence de marche $\Delta$ en fonction de l'angle $\theta$ d'inclinaison de la lame est donnée par l'expression :

$$
\Delta=e_{0}\left(n^{2}-\sin ^{2} \theta\right)^{1 / 2}-e_{0} \cos \theta .
$$

Cette expression montre qu'il est pratiquement impossible de réaliser des variations de $\Delta$ linéaires par rapport au temps. Ce type de modulation a l'inconvénient de donner un signal dont la fréquence n'est pas fixe au cours de l'oscillation de la lame compensatrice et de plus, il existe un régime transitoire quand la lame compensatrice change de sens (Fig. 2).

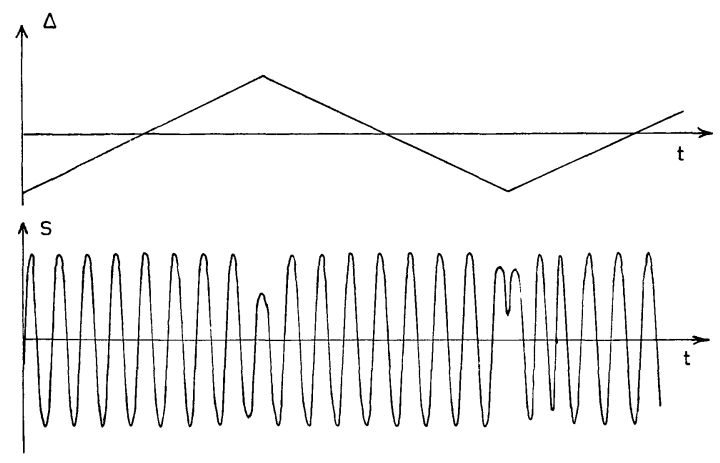

FIG. 2. - Modulation incohérente par une compensatrice oscillante.

Ce procédé de modulation nécessite l'emploi d'un filtre électronique relativement large pour diminuer l'amplitude du «bruit de modulation » trop important. En détection simple le rapport signal/bruit baisse et l'on perd une partie du gain de luminosité fourni par le SISAM.

Des essais de détection synchrone ont déjà été faits en utilisant une partie du flux lumineux comme signal de référence, afin de réduire le «bruit de modulation» et d'obtenir une détection linéaire [5], [6]. 
Les systèmes réalisés ne semblent pas très commodes d'emploi (nécessité de deux détecteurs) et des variations de phase entre les deux parties du faisceau lumineux sont toujours possibles au cours de la rotation des réseaux.

Dans le montage à double passage (Fig. 1b) que nous avons réalisé, la modulation était obtenue par un déplacement linéaire alternatif d'un des miroirs servant au double passage. Il était avec ce système plus facile d'obtenir un signal à fréquence fixe mais il subsistait toujours des régimes transitoires.

C'est pourquoi, nous avons étudié et réalisé une modulation cohérente permettant d'effectuer une détection synchrone. Le principe de la modulation cohérente est en principe assez simple: il suffit de faire vibrer parallèlement à lui-même un des miroirs de l'interféromètre avec une amplitude égale à un huitième de la longueur d'onde de réglage, à partir de la différence de marche moyenne $\Delta_{0}=(2 K+1) \lambda / 4$. Mais il faut absolument que $\Delta_{0}$ reste fixé à sa valeur (aussi proche que possible de $\Delta_{0}=\lambda / 4$ ) au cours de la rotation des réseaux, et en tenant compte en plus des dilatations possibles de l'appareil. Un réglage parfait et qui ne varierait pas étant impossible à réaliser, il faut asservir la différence de marche entre les deux bras de l'interféromètre.

Le SISAM étant réglé et éclairé par une onde plane de nombre d'onde $\sigma$, l'intensité lumineuse à la sortie de l'interféromètre a pour expression:

$$
I=I_{0}(1+\cos 2 \pi \sigma \Delta) .
$$

Lorsque l'on fait vibrer avec l'amplitude $a / 2$ un des miroirs à la fréquence $\omega / 2 \pi$, la différence de marche a pour expression :

$$
\Delta=\Delta_{0}+a \cos \omega t,
$$

d'où l'intensité lumineuse

$$
I=I_{0}\left\{1+\cos \left|2 \pi \sigma\left(\Delta_{0}+a \cos \omega t\right)\right|\right\} .
$$

Le développement en série de Fourier du signal électrique $S$ fourni par le détecteur donne, en se limitant au fondamental et au premier harmonique :

$$
S=A \sin 2 \pi \sigma \Delta_{0} \cos \omega t+B \cos 2 \pi \sigma \Delta_{0} \cos 2 \omega t .
$$

Nous voyons que le signal à la fréquence $\omega / 2 \pi$ est maximum quand $\Delta_{0}=(2 k+1) \lambda / 4$. Le signal à la fréquence $\omega / \pi$ est alors nul. Lorsque $\Delta_{0}$ s'écarte de cette valeur, le signal à la fréquence double n'est plus nul et sa phase varie de $\pi$ quand on passe de $\Delta_{0}-\varepsilon$ à $\Delta_{0}+\varepsilon$.

On peut donc, en réalisant une détection synchrone sur le premier harmonique, obtenir un signal d'erreur qui permet d'asservir la différence de marche entre les deux bras du SISAM. L'ensemble électronique (Fig. 3) comprend essentiellement un oscillateur sinusoïdal $(400 \mathrm{~Hz})$ fournissant le signal qui commande la vibration du miroir $\mathrm{M}_{2}$ et le signal de référence pour la détection synchrone du signal à la fréquence fondamentale. Cet oscillateur est également suivi d'un

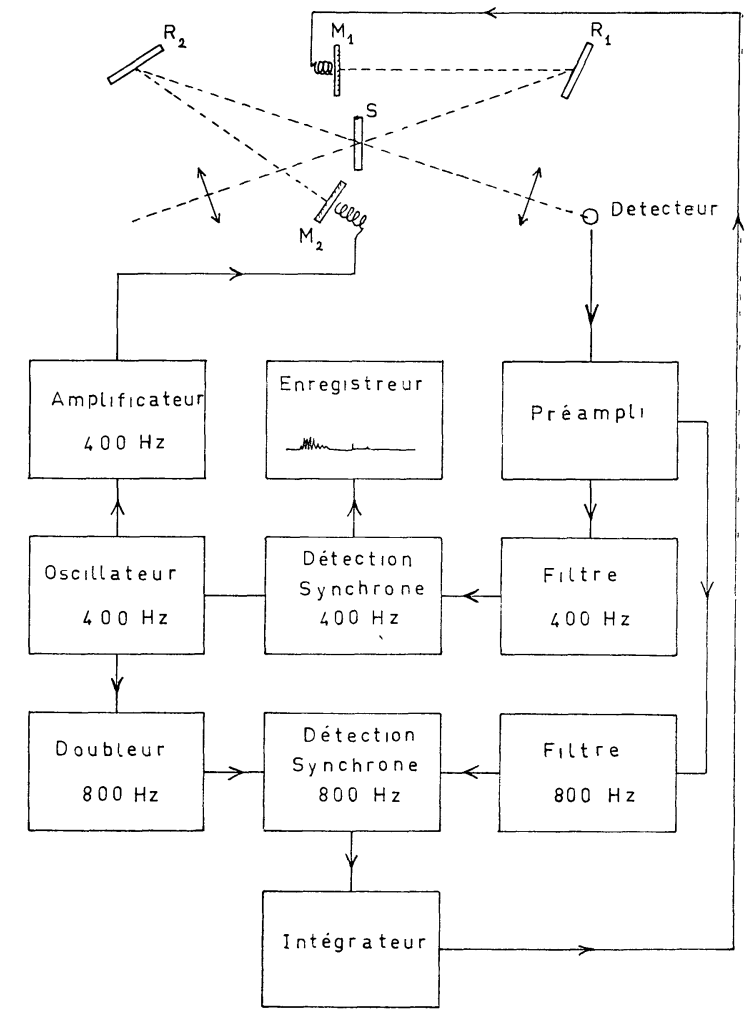

Fig. 3. - Ensemble électronique assurant une modulation cohérente, une détection synchrone et un asservissement de la différence de marche.

doubleur de fréquence, qui donne le signal de référence pour la détection synchrone du premier harmonique. Le signal d'erreur est envoyé sur un intégrateur qui fixe la position du miroir mobile $\mathrm{M}_{1}$. Ce dernier est monté sur un parallélogramme déformable. Une force de rappel mécanique et un amortissement visqueux évitent toute vibration de cet équipage mobile.

Les résultats obtenus sont illustrés par deux enregistrements de la branche $\mathrm{Q}$ du méthane à 1,6 micron (montage à double passage symétrique). La figure 4 présente le spectre tracé en utilisant un enregistreur potentiométrique. Cet enregistrement a duré $15 \mathrm{mn}$, il ne présente plus aucun bruit de modulation. Le bruit apparent est dû essentiellement aux fluctuations d'in-

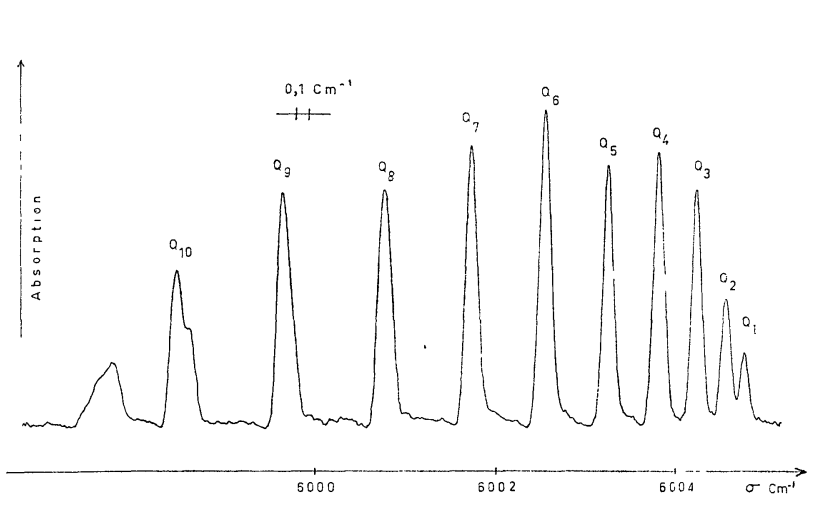

FIG. 4. - Enregistrement en détection synchrone. 
dice de l'air dans l'interféromètre qui pour l'instant n'est pas sous vide.

La figure 5 montre le même spectre enregistré à l'oscilloscope en $15 \mathrm{~s}$. Nous voyons que le gain en

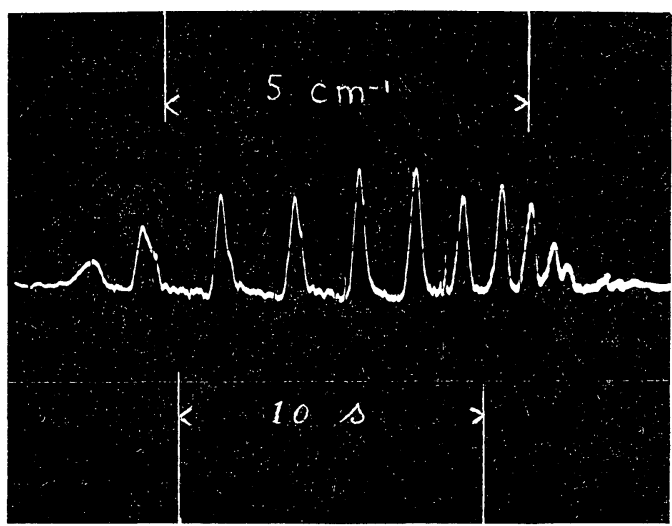

Fig. 5. - Enregistrement rapide du même spectre que celui de la figure 4 en vitesse rapide. rapport signal/bruit peut être exploité en gain de temps lorsque l'on dispose d'une source suffisamment intense comme c'est le cas généralement pour l'étude des spectres d'absorption.

Dans le montage asymétrique, le système qui vient d'être décrit présentera un grand intérêt, car il est alors très important que $\Delta_{0}$ soit très proche de la valeur zéro et ne s'en écarte pas.

Le SISAM présentait jusqu'ici l'avantage d'avoir une grande luminosité et une résolution élevée, mais le gain de luminosité, très important par rapport aux spectromètres classiques, n'était pas pleinement utilisé puisque la détection n'était pas linéaire, ce qui est nécessaire pour effectuer des mesures d'intensité, et que le bruit de modulation conduisait à travailler avec des constantes de temps rarement inférieures à la seconde.

L'appareil qui vient d'être réalisé permet de profiter maintenant de façon très pratique de tous les avantages de luminosité et de résolution du SISAM.

\section{Bibliographie}

[1] Connes (P.). - J. Physique Rad., 1956, 17, 1.

[2] Connes (P.). - Thèse Paris, 1957.

[3] Dupre (J.), Pinson (P.), Meyer (C.), Barchewitz (P.). - Appl. Opt., 1971, 10, 1177.
[4] Pinson (P.), Dupre (J.), Meyer (C.). - C. R. Acad. Sci. Paris, 1971, 273, 587.

[5] Graner (G.). - Thèse Paris, 1965, § 4-5, p. 35

[6] Verges (J.). - Thèse Paris, 1969, § 1-7, p. 13. 